\title{
A new species of Mycena sect. Exornatae and some taxonomic observations
}

\section{Aravindakshan DM and Manimohan P*}

\author{
Department of Botany, University of Calicut, Kerala, 673 635, India
}

Aravindakshan DM, Manimohan P 2013 - A new species of Mycena sect. Exornatae and some taxonomic observations. Mycosphere 4(1), 146-150, Doi 10.5943/mycosphere/4/1/9

Mycena snigdha sp. nov. is described from Kerala state, India and is assigned to sect. Exornatae. Comprehensive description, photographs, and comparisons with phenetically similar species are provided. The wide, loop-like clamp connections of $M$. snigdha are remarkably similar to those of species of Mycena sect. Ingratae and this observation reveals that loop-like clamp connections can no longer be used to differentiate sect. Ingratae from sect. Exornatae and supports a recent treatment of sect. Ingratae as a synonym of section Exornatae. Within sect. Exornatae, however, this feature seems to be useful in species segregation. Mycena fumosa, an Australian species currently placed in sect. Sacchariferae, is considered as belonging to sect. Exornatae.

Key words - Agaricales - Basidiomycota - biodiversity - Mycenaceae - taxonomy

\section{Article Information}

Received 11 February 2013

Accepted 24 February 2013

Published online 28 February 2013

*Corresponding author: P Manimohan - e-mail - pmanimohan@gmail.com

\section{Introduction}

Section Exornatae Maas Geest. of the genus Mycena (Pers.) Roussel (Mycenaceae, Agaricales, Basidiomycota) comprises species with medium-sized basidiomata with a discoid stipe base, gelatinized pileipellis composed of hyphae with thorn-like excrescences, nongelatinised stipitipellis and amyloid basidiospores. The section was proposed by Maas Geesteranus (1982) to accommodate $M$. boninensis (Berk. \& Curt.) Sing., a species that was later reduced to the synonymy of $M$. chlorophos (Berk. \& M.A. Curtis) Sacc. (Maas Geesteranus 1992). Recently Desjardin et al (2010) reduced sect. Ingratae Maas Geest. to synonymy with sect. Exornatae as loop-like clamp connections on the pileipellis hyphae of the former, the only difference between the two sections, are seen among several other sections of Mycena and hence was not considered as a character significant enough to differentiate sections. Although it is a small group comprising about 7-8 species, these species have been recorded from places as far apart as Australia, Belize, Brazil, Dominican Republic, Jamaica, Japan (Bonin Islands), India, Indonesia, Madagascar, Malaysia, Papua New Guinea, Puerto Rico, Sri Lanka and USA (Florida) in the pan-tropical belt (Desjardin et al. 2010, Aravindakshan \& Manimohan 2012). During the course of our studies on mycenas of Kerala State, India, we came across a hitherto undescribed species that belong in this section and it is described and discussed here.

\section{Methods}

Conventional morphology-based taxonomic methods were employed for this study. Microscopic observations were made on material stained with $1 \%$ aqueous solution of 
Congo red and mounted in $3 \%$ aqueous $\mathrm{KOH}$. Melzer's reagent was used to observe whether the spores and tissues were amyloid. For evaluation of the range of spore-size, twenty basidiospores each from one specimen of each collection cited were measured. Colour codes used in the descriptions are from Kornerup \& Wanscher (1978). Two of the examined collections cited, including the holotype, are deposited at the Kew (Mycology) herbarium and their Kew accession numbers (e.g., $\mathrm{K}(\mathrm{M}) 178335$ ) are provided. The collections cited without Kew accession numbers are in the personal herbarium of the second author. The concept of Mycena sect. Exornatae followed is that of Maas Geesteranus (1992) as modified by Desjardin et al. (2010).

\section{Results}

\section{Taxonomy}

\section{Mycena snigdha Aravind. \& Manim., sp. nov.}

MycoBank MB 803282

gelatinous.

Etymology - snigdha (Sanskrit),

Characterised by gelatinous pileipellis composed of hyphae with much branched side branches that are covered with thorn-like excrescences, non-gelatinized stipitipellis, fusoid cheilo- and caulocystidia, amyloid basidiospores and prominent basal disc of stipe. Differing from Mycena discobasis in having non-luminescent basidiomata, cheilocystidia often with a long apical prolongation, hyphae of the pileipellis with prominent thorn-like excrescences and looplike clamp connections and a pileal margin composed entirely of acanthophysoid terminal cells.

Basidiomata very small, delicate. Pileus 1.5-4.5 mm diam., 0.75-1 mm high, conicoparabolic to hemispherical when young, becoming plano-convex with a centrally flattened depression; surface brown (5E4) or greyish brown (7E3) at the centre and marble white (5B2) or brownish grey (7D2) towards margin when young, becoming brown (5E4) or greyish brown $(5 \mathrm{D} 3,7 \mathrm{~F} 3)$ at the centre and brownish grey $(5 \mathrm{C} 3,7 \mathrm{C} 2)$ towards margin with age, translucent-striate when young, becoming nearly sulcate towards margin, finely pruinose, dry; margin slightly incurved and entire when young, becoming straight and finely torn with age. Lamellae 13-19 reaching the stipe, free or attached to a slight collar, pale grey or offwhite, 0.5-1 mm thick, subclose, with lamellulae of 2 lengths; edge finely torn under a lens, paler than the sides. Stipe $2-10.3 \times$ 0.25-1 mm, central, terete, almost equal when young, becoming slightly tapering towards apex with age, hollow; surface translucent, greyish to white when young, becoming marble white (5B2) with age, finely pubescent all over when young, becoming glabrous towards apex, more or less densely pubescent towards base with age; base discoid, of radiating mycelium, hairy. Context not conspicuous. Odour and taste not distinctive.

Basidiospores (6) 7-9 (10.5) × 4.5-6 $(7.628 \pm 0.595 \times 4.8625 \pm 0.29) \mu \mathrm{m}, \mathrm{Q}=1.3-$ 1.85, $\mathrm{Qm}=1.57$, ellipsoid, thin-walled, hyaline, smooth, strongly amyloid. Basidia (10) 14-20 × 8.5-11 $\mu \mathrm{m}$, narrowly clavate, bearing 4 sterigmata up to $5.5 \mu \mathrm{m}$ long. Lamella-edge sterile. Cheilocystidia crowded, 18-40 × 9-20 $\mu \mathrm{m}$, fusoid or broadly fusoid or vesiculose, thin- to slightly thick-walled $(0.5 \mu \mathrm{m})$, hyaline, often rostrate or with a very fragile, often curved, filamentous, rarely furcate protrusions $(2-16 \times 3-4 \mu \mathrm{m})$. Pleurocystidia none. Lamellar trama regular to subregular; hyphae 2-29 $\mu \mathrm{m}$ wide, thin-walled, hyaline to pale greyish, faintly vinoid to moderately vinoid in Melzer's reagent. Subhymenium ramose. Pileus trama subregular; hyphae 3-34 $\mu \mathrm{m}$ wide, thin-walled, hyaline to pale greyish, faintly to moderately vinoid in Melzer's reagent. Pileipellis an epicutis; hyphae 1.5-13 (20) $\mu \mathrm{m}$ wide, thin-walled, hyaline, with numerous lateral thorn-like protrusions (3-17 $\mu \mathrm{m}$ long), embedded in a gelatinous matrix, with prominent, loop-like clamp-connections. Pileocystidia $34.5-78 \times 11-34.5 \mu \mathrm{m}$, clavate to narrowly clavate or obovoid, thin-walled, hyaline, with simple, cylindrical excrescences $(0.5-6.5 \times 0.5-1.5 \mu \mathrm{m})$. Terminal cells at pileus margin $16-51.5 \times 12-26.5 \mu \mathrm{m}$, clavate or ellipsoid or fusoid, thin-walled, hyaline, with simple, cylindrical excrescences (0.5-2.5 $\times 0.5-1 \mu \mathrm{m})$. Stipitipellis a cutis of smooth hyphae; hyphae $1.5-8 \mu \mathrm{m}$ wide, thin- to very slightly thick-walled $(0.25 \mu \mathrm{m})$, hyaline. 
Mycosphere Doi 10.5943/mycosphere/4/1/9

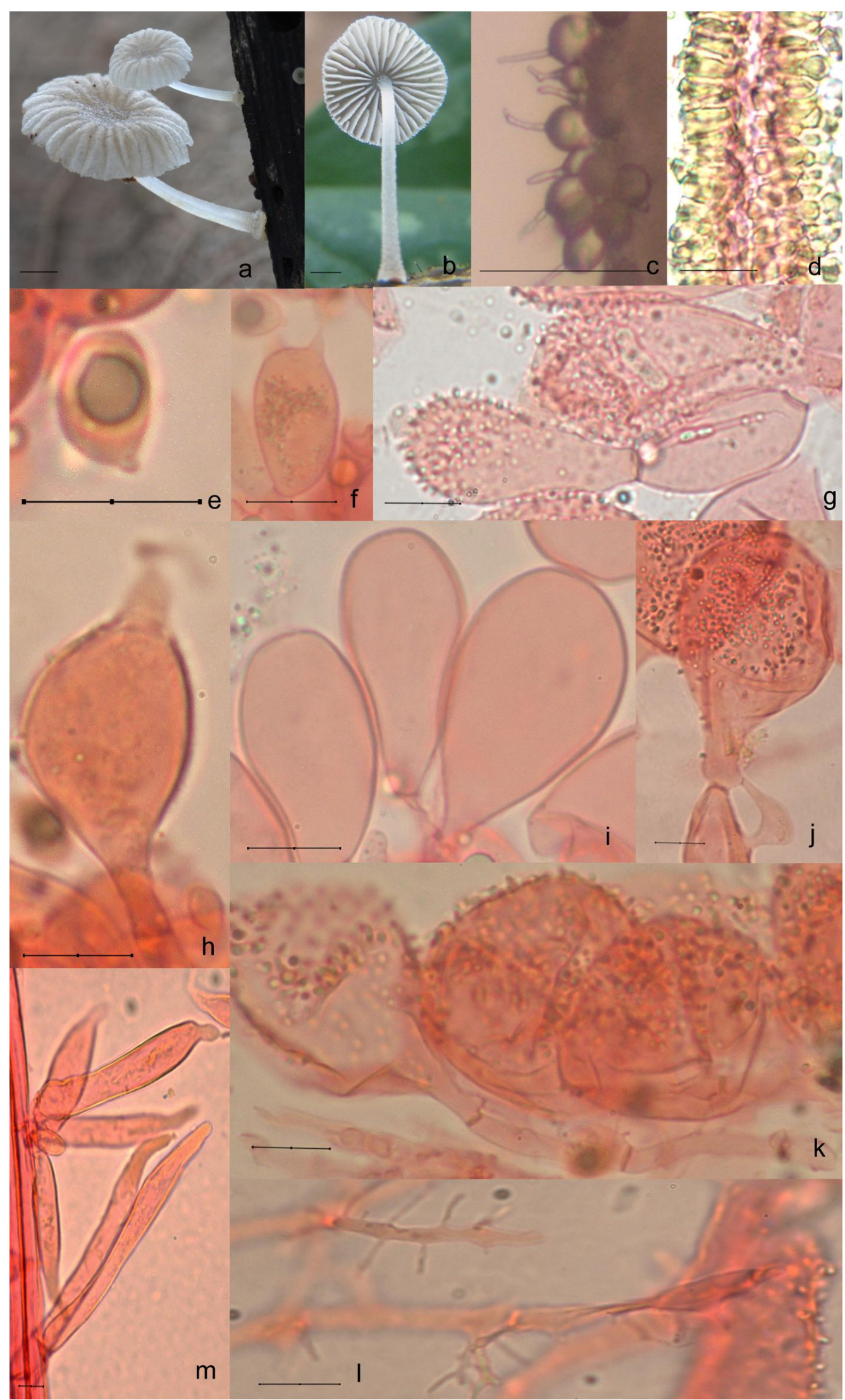

Fig. 1 a-m - Mycena snigdha. a, b Basidiomata. c Close-up of lamella-edge. d Lamellar trama stained with Melzer's reagent. e Basidiospore. f Basidium. g Cells of the pileus margin. h, i cheilocystidia. $\mathbf{j}$ Pileocystidium with loop-like clamp connection. $\mathbf{k}$ Hyphae of the pileipellis with pileocystidia. $\mathbf{l}$ Thorn-like excrescences of the pileipellis hyphae. $\mathbf{m}$ Caulocystia. - Bars $=1 \mathrm{~mm}$ for basidiomata and $10 \mu \mathrm{m}$ for micro-structures. 
Caulocystidia (16) $27-146 \times 7-23$ (27) $\mu \mathrm{m}$, ellipsoid, oblong or cylindrical or narrowly fusoid or lageniform, thin-walled, hyaline, pedicellate or not, occasionally mucronate or with an apical protrusion $(4-10 \times 1-2 \mu \mathrm{m})$. Stipe trama strongly vinoid in Melzer's reagent. Clamp connections seen on all hyphae.

On the bark of standing trees usually among moss and also on decaying twigs, scattered, June-July.

type locality.

Distribution - known only from the

Material examined - INDIA, Kerala State, Malappuram District, Calicut University Campus: 24 July 2007, D.M. Aravindakshan DM184 (K(M)178336); 4 July 2010, DM Aravindakshan DM445; 8 June 2011, D.M. Aravindakshan DM523; 28 July 2011, D.M. Aravindakshan DM528 (K(M)178335, holotype).

\section{Discussion}

The gelatinous pileipellis composed of hyphae with much branched side branches that are covered with thorn-like excrescences, the non-gelatinized stipitipellis, the fusoid cheiloand caulocystidia, the amyloid basidiospores and the prominent basal disc of stipe indicate that M. snigdha is best placed in sect. Exornatae. Within sect. Exornatae, it is closely allied with $M$. discobasis Métrod sharing several macro- and microscopic features. However, unlike $M$. discobasis, $M$. snigdha has non-luminescent basidiomata, smaller pilei (less than $5 \mathrm{~mm}$ broad), cheilocystidia often with a long apical prolongation, hyphae of the pileipellis with prominent thorn-like excrescences and loop-like clamp connections and a pileal margin composed entirely of acanthophysoid terminal cells. Also, no bioluminescence was observed when fresh basidiomata of $M$. snigdha with intact basal mycelium and supporting bark were observed in a dark room. Mycena chlorophos differs from the present species in having larger, luminescent basidiomata, strong nitrous odour, numerous lamellae (18-32), large basidia and cheilocystidia, narrower pileocystidia, smaller caulocystidia without mucronate or apical protrusion and normal clamp connections. Additionally, the pileus and lamellae of $M$. chlorophos show bright bluish green- luminescence. Mycena deeptha Aravind. \& Manim, an Indian species, differs in having luminescent mycelium, detersile elements over the primordium, and caulocystidia with a very long protrusion.

Mycena chlorinosma Singer differs in having larger basidiomata, chlorine odour, numerous lamellae (c. 40), reddish brown to dark red-brown stipe base, larger cheilocystidia without protrusions and smaller caulocystidia without protrusions. Mycena margarita (Murrill) Murril differs from the present species in having somewhat larger basidiomata, adnexed lamellae, yellowish-buff staining at the stipe base, cheilocystidia and caulocystidia without protrusions, narrow pileus marginal cells and luminescent basidiomata (nonluminescent in some populations). Mycena rhenana Maas Geest. \& Winterh., originally assigned to sect. Basipedes (Fr.) Quel. that according to Desjardin et al. (2007) is best placed in sect. Exornatae, lacks cheilocystidia and has pileus marginal cells of different morphology. Mycena interrupta (Berk.) Sacc., currently placed in sect. Cyanocephalae Singer but best assigned to sect. Exornatae (Desjardin et al. 2010) has blue pigmented basidiomata, filamentous caulocystidia and spinulose cheilocystidia.

Mycena fumosa Grgur., an Australian species that was originally described as a variety of $M$. interrupta and currently placed in sect. Sacchariferae Kühner (Grgurinovic 2002) has several features similar to those of the present species including gelatinized pileipellis hyphae with prominent loop-like clamp connections. That species, however, has larger basidiomata and cystidia lacking apical prolongation. Also, the thorn-like excrescences on the pileipellis hyphae are not well developed. We consider Mycena fumosa, with its gelatinized pileipellis with ornamented hyphae and loop-like clamp connections, fusoid cystidia and discoid stipe base, as belonging to sect. Exornatae.

The wide, loop-like clamp connections on the pileipellis hyphae of the present species are remarkably similar to those of species earlier assigned to Mycena sect. Ingratae (Mycena chlorinosma and M. subepipterygia Murrill, currently considered as synonyms of M. margarita). The present observations reveal 
that loop-like clamp connections can no longer be used to differentiate sect. Ingratae from sect. Exornatae and support the merger of the two sections by Desjardin et al. (2010). Within sect. Exornatae, however, this feature seems to be useful in species segregation.

\section{References}

Aravindakshan DM, Kumar TKA, Manimohan P. 2012 - A new bioluminescent species of Mycena sect. Exornatae from Kerala State, India. Mycosphere 3(5), 556-561.

Desjardin DE, Capelari M, Stevani C. 2007 Bioluminescent Mycena species from São Paulo, Brazil. Mycologia 99 (2), 317-331.

Desjardin DE, Perry BA, Lodge DJ, Stevani C,
Nagasawa E. 2010 - Luminescent Mycena: new and noteworthy species. Mycologia 102 (2), 459-477.

Grgurinovic CA. 2002 - The Genus Mycena in South-Eastern Australia. Fungal Diversity Press, Hong Kong.

Kornerup A, Wanscher JH. 1978 - Methuen Handbook of Colour. 3rd Ed. Eyre Methuen, London.

Maas Geesteranus RA. 1982 - Studies in Mycenas 72. Berkeley's fungi referred to Mycena - 2. Proceedings van de Koninklijke Nederlandse Akademie van Wetenschappen (Section C) 85, 527-539.

Maas Geesteranus RA. 1992 - Mycenas of the Northern Hemisphere II. Conspectus of the Mycenas of the Northern Hemisphere. North-Holland, Amsterdam. 